\title{
Higgs physics at CMS
}

\author{
Milos Dordevic on behalf of the CMS Collaboration ${ }^{1, *}$ \\ ${ }^{1}$ Vinca Institute of Nuclear Sciences, University of Belgrade
}

\begin{abstract}
The most recent results of a Higgs boson production and properties from the CMS Collaboration using the LHC Run 1 and Run 2 data are reported. These include analyses of a Higgs boson decaying to a pair of photons, four leptons via $\mathrm{Z}$ boson pair decays and the associated production of a Higgs boson with top quark pair, predicted by the Standard Model (SM). The studies of a Higgs boson decays to a pair of bottom quarks, a pair of tau leptons and a charm quark pair are also presented. The Higgs boson production via vector boson fusion (VBF) and decaying to invisible particles is reported here as well. The analysis of a Higgs boson decay to a pair of muons is also presented. The study of a Higgs boson pair production at $13 \mathrm{TeV}$ is performed as well and projections of a Higgs boson self couplings together with the couplings to other particles at the HL-LHC are made.
\end{abstract}

\section{Introduction}

The Higgs boson was discovered in 2012 at CERN by the ATLAS and CMS Collaborations [1-3], with mass measured to be around $125 \mathrm{GeV}$ and precise measurements of its spin-parity, width, boson and fermion couplings were pursued ever since the discovery. All the performed measurement have turned out to be consistent with the prediction of the SM. With the full LHC Run 2 data available, the focus of the Higgs boson studies shifted from the Higgs boson discovery also to the precision measurements of the Higgs boson couplings and properties. The yet not accessible decay modes are being studied, the Higgs boson anomalous couplings are being searched for and a large available datasets have enabled to study also the differential cross sections. The recent results of the Higgs boson studies by the CMS Collaboration [4] are presented in this report.

\section{Higgs boson decay to two photons}

The analysis of a Higgs boson decaying to two photons was performed targeting the gluongluon fusion ( $\mathrm{ggF}$ ) and the vector boson fusion (VBF) production modes, using the data recorded by the CMS experiment in 2016 and 2017 [5]. The simplified template cross section (STXS) framework [6] that minimizes a theory dependence of the Higgs boson measurement was used in this analysis. The extensive usage of Boosted Decision Trees (BDT) was applied and dedicated BDTs were trained for the photon and vertex identification, signal extraction and the event categorization. The signal plus background fit was performed in all analysis categories. The fitted diphoton invariant mass spectrum for the sum of all categories, each

*e-mail: milos.dordevic@cern.ch 
weighted by the ratio of the number of signal over signal plus background events, $S /(S+B)$, is presented in Fig. 1. The measured cross section, normalized to the corresponding SM prediction, targeting the $g g F$ and VBF production modes is found to be: $\sigma_{g g H} / \sigma_{g g H}^{S M}=1.15_{-0.15}^{+0.15}$ and $\sigma_{q q H} / \sigma_{q q H}^{S M}=0.8_{-0.3}^{+0.4}$. The results of a seven parameter fit performed using the STXS framework are presented in Fig. 2, each showing a good agreement with the SM prediction, within the uncertainties.

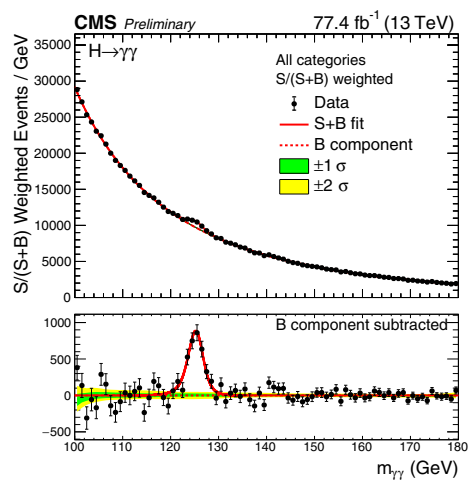

Figure 1. Signal plus background model fit to the diphoton invariant mass spectrum for the sum of all categories. The bottom plot shows the residuals after the background component is subtraction [5].

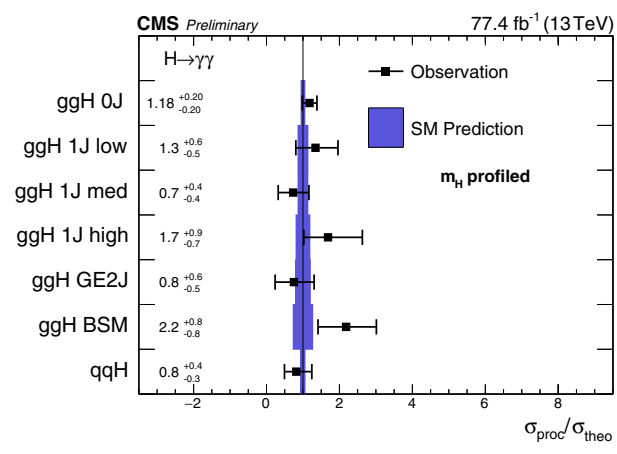

Figure 2. The results of a sevenparameter fit in the STXS framework. Cross section ratios are shown with approximate $68 \%$ CL intervals (black points), and compared to the SM expectations and uncertainties (blue bands) [5].

\section{Higgs boson decay to four leptons}

The decay of a Higgs boson to four leptons, being either electrons or muons, via production of a $\mathrm{Z}$ boson pair has a clean experimental signature with a large signal to background ratio, due to an excellent lepton momentum resolution. It also provides a possibility for a precise measurement of the Higgs boson mass and width.

\subsection{Measurement of the Higgs boson mass}

The Higgs boson mass was measured with the 2016 data recorded by the CMS experiment, using its decay to four leptons via production of a $\mathrm{Z}$ boson pair [7]. The reconstructed invariant mass of the four lepton system is presented in Fig. 3. This analysis applies matrix element discriminants in order to enhance the signal sensitivity. The three-dimensional fit, taking account the four lepton invariant mass, a per-event estimate of the mass resolution and a kinematic discriminant variable as the inputs was performed. This lead to the measured combined value of the Higgs boson mass of $m_{H}=125.26 \pm 0.20$ (stat.) \pm 0.08 (syst.) GeV. The likelihood scan versus the Higgs boson mass, while profiling the signal strength modifier $\mu$ along with all other nuisance parameters, is shown in Fig. 4 . The systematic uncertainty in the mass measurement is totally dominated by the uncertainty in the lepton momentum scale.

\subsection{Measurement of the Higgs boson width}

The Higgs boson width was studied in the four lepton final state using the combination of the Run 1 data with the Run 2 data recorded by the CMS experiment in 2016 and 2017 [8]. 


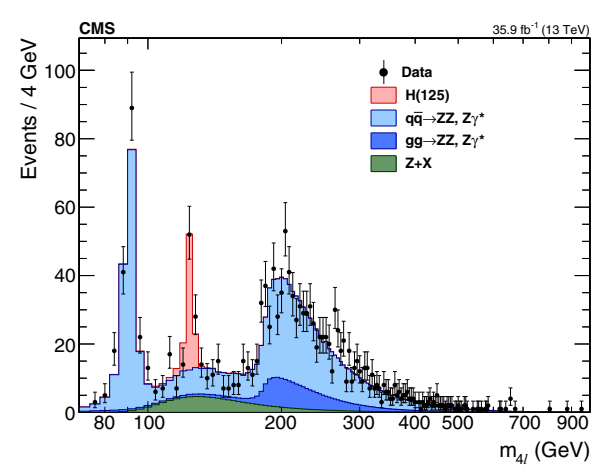

Figure 3. Distribution of the reconstructed four-lepton invariant mass $m_{4 l}$ in the full mass range [7].

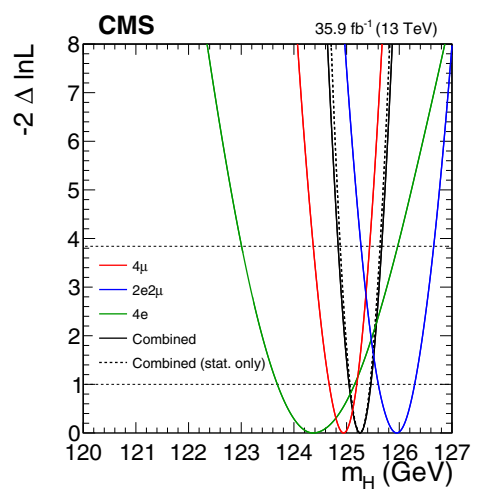

Figure 4. 1D likelihood scans as a function of the mass for a different final states and a combination of all final states for the 3D mass measurement [7].

The kinematic information from the decay particles and the associated jets are combined using matrix element techniques in order to identify the production mechanism and to increase the sensitivity to the Higgs boson couplings in both production and decay. The Higgs boson width was measured using the off-shell production method that relies on the relative ratio of the off-shell to the on-shell measurement. The width constraints are shown in Fig. 5 which represents the observed (solid) and expected (dashed) likelihood scans. The constraints on the anomalous HVV couplings are found to be consistent with the SM expectation in both on-shell and off-shell regions. With the assumption of a coupling structure similar to that in the SM, the Higgs boson width is constrained to be $3.2_{-2.2}^{+2.8} \mathrm{MeV}$, while the expected constraint based on simulation is $4.1_{-4.0}^{+5.0} \mathrm{MeV}$. This width measurement is significantly better than using the on-shell method alone, that is approximately $1 \mathrm{GeV}$ [7-9]. No anomalous HVV interactions were observed.

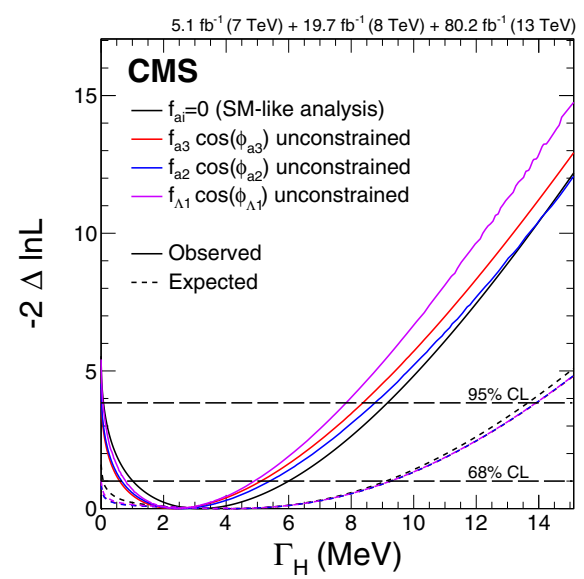

Figure 5. Observed (solid) and expected (dashed) likelihood scans of $\Gamma_{H}$. Results of the combined Run 1 and Run 2 data analyses, with the 2015 data included in the on-shell case. The dashed horizontal lines show the $68 \%$ and $95 \%$ CL regions [8]. 


\subsection{Differential and fiducial cross sections}

The differential and fiducial cross sections were measured in the four lepton final state using the full Run 2 dataset [11]. The cross section for the Higgs boson decay to four leptons in a fiducial region closely matching the experimental selection of the leptons is measured to be $\sigma=2.73_{-0.22}^{+0.23}(\text { stat. })_{-0.19}^{+0.24}$ (syst.) fb at $m_{H}=125.09 \mathrm{GeV}$, compared to the SM prediction of $2.76 \pm 0.14 \mathrm{fb}$. The inclusive fiducial cross section in different final states and their combination is shown in Fig. 6. The differential cross sections as a function of the $p_{T}$ and rapidity of the Higgs boson, the number of associated jets and the $p_{T}$ of the leading associated jet are determined. In the Fig. 7 the differential cross section as a a function of the $p_{T}$ is presented. All results are consistent with the expectations for the SM Higgs boson, within the uncertainties.

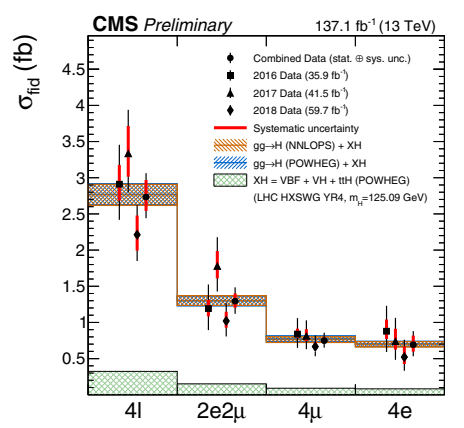

Figure 6. The measured inclusive fiducial cross section in different final states [11].

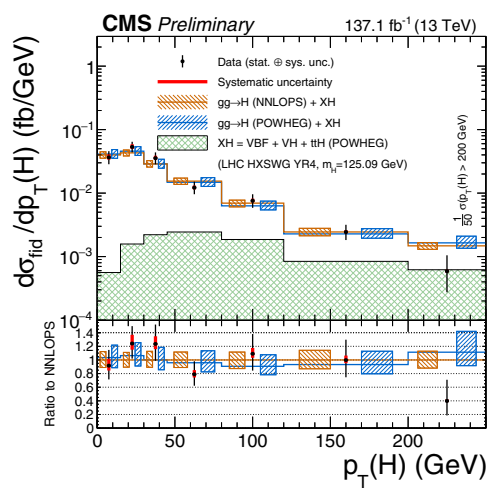

Figure 7. The results of the differential cross section measurement for $p_{T}[11]$.

\section{Higgs boson production with top quark pair}

The observation of a Higgs boson production in association with a top quark-antiquark pair $(\mathrm{ttH})$ is reported in [12], where the analyses performed with data recorded at 7, 8 and 13 $\mathrm{TeV}$ were combined. This analysis of $\mathrm{ttH}$ production allows for a direct measurement of the top quark Yukawa coupling $\left(y_{t}\right)$. The results of a statistically independent search for Higgs boson production associated to a top quark pair and decaying to a pair of $\mathrm{W}$ bosons, $\mathrm{Z}$ bosons, photons, tau leptons or bottom quark jets are combined to maximize sensitivity. The presence of a ttH signal is assessed by performing a simultaneous fit to the data from the different decay modes. The distribution of a $\log _{10}(S / B)$ is shown in Fig. 8, where the $S$ and $\mathrm{B}$ are the expected post-fit signal (with $\mu_{t t H}=1$ ) and background yields, respectively, in each bin of the distributions considered in the combination. The value of a test statistic $\mathrm{q}$ as a function of the $\mu_{t t H}$ is shown in Fig. 9, with $\mu_{t t H}$ based on the combination of decay modes described above for the combined fit. The observed significance is 5.2 standard deviations, while the expected significance for a SM Higgs boson with a mass of $125.09 \mathrm{GeV}$ is 4.2 standard deviations.

The $\mathrm{ttH}$ analysis with multilepton final states, including Higgs boson decays to a pair of $\mathrm{W}$ bosons, $\mathrm{Z}$ bosons and tau leptons is performed also with 2017 data and reported in [13]. The analysis used BDT and matrix-element discriminant methods for signal extraction, also combined in a two-dimensional approach (2D BDT), trained against $t \bar{t}$ and $t \mathrm{VV}(\mathrm{W} / \mathrm{Z})$ backgrounds, where the $2 \mathrm{D}$ space is partitioned using recursive methods, maximizing signal sensitivity. An excess of events is observed in the highest bins of the BDT distribution, 


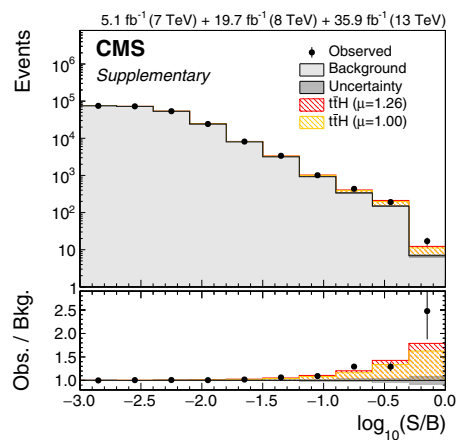

Figure 8. Distribution of the events as a function of the decimal logarithm of $\mathrm{S} / \mathrm{B}$, where the $\mathrm{S}$ and $\mathrm{B}$ are the expected post-fit signal (with $\mu_{t t H}=1$ ) and background yields, respectively, in each bin of the distributions in this combination [12].

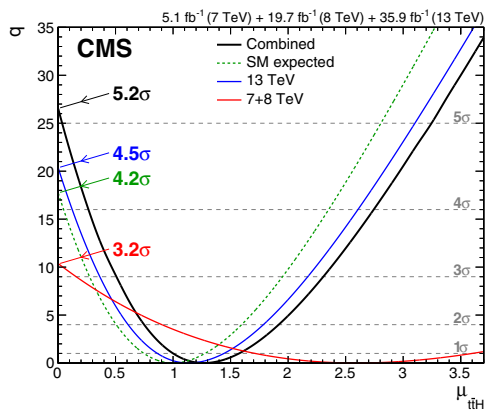

Figure 9. The test statistic q, described in the text, as a function of $\mu_{t t H}$ for all decay modes at 7+8 TeV and at $13 \mathrm{TeV}$, separately, and for all the decay modes at all the system of center of mass energies [12].

as shown in Fig. 10, corresponding to the observed significance of 3.2 standard deviations, with the expected significance of 4.0 standard deviations. The analysis of a Higgs boson decaying to a pair of bottom quarks is also updated in [14], yielding with observed (expected) signal significance of 3.9 (3.5) standard deviations. These results represent a first evidence of $\mathrm{ttH}(\mathrm{bb})$ production, following also the updated b-tagger and the optimized analysis. The analysis of the $\mathrm{ttH}$ production where a Higgs boson decays to a pair of photons is also performed using the 2017 data [14], yielding an observed signal significance of 4.1 and expected significance of 2.7 standard deviations.

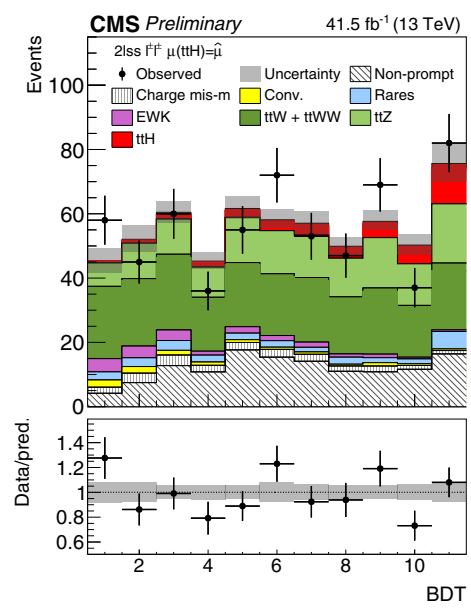

Figure 10. The distribution of the discriminating observable used for the signal extraction in the 2lss categories [13].

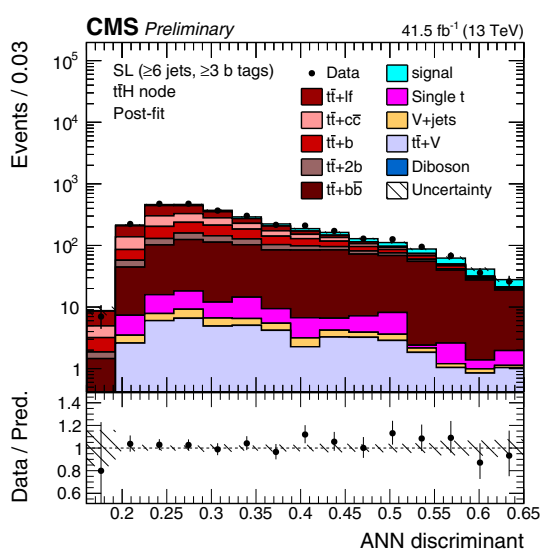

Figure 11. The final discriminant shape in the categories with high sensitivity in the semi-leptonic channel after a fit to data [14]. 


\section{Higgs boson decay to bottom quarks}

The analysis of a Higgs boson decay to bottom quarks, allowing to test the top quark Yukawa coupling to the down-type quark, is presented in [15]. The most sensitive Higgs boson production process is the associated production to a vector boson, being either a $\mathrm{W}$ or $\mathrm{Z}$ boson. Depending on the decay of vector bosons, the final states with either 0,1 or 2 leptons, together with a two bottom quark jets are considered in this analysis. The most important backgrounds to this process are the $\mathrm{V}+$ jets, $t \bar{t}$ and single top quark production and the QCD multijet process. A deep neural network (DNN) discriminant was used to tag the bottom quark jets. The dijet mass resolution is improved using the DNN regression method. The signal is extracted by fitting the DNN score. An alternative to fitting the DNN score is to fit the m(jj) distribution, which results in less sensitivity but enables a more direct visualization of the Higgs boson signal, as shown in Fig. 12. The best fit to the signal strength in the individual channels and their combination is presented in Fig. 13. Combining this result with the previous measurements of this decay by the CMS Collaboration, in the events where the Higgs boson is produced through gluon fusion, vector boson fusion, or in an association with top quarks, the observed (expected) significance is 5.6 (5.5) standard deviations. This constitutes an observation of the Higgs boson decay to a bottom quark pair, by the CMS Collaboration.

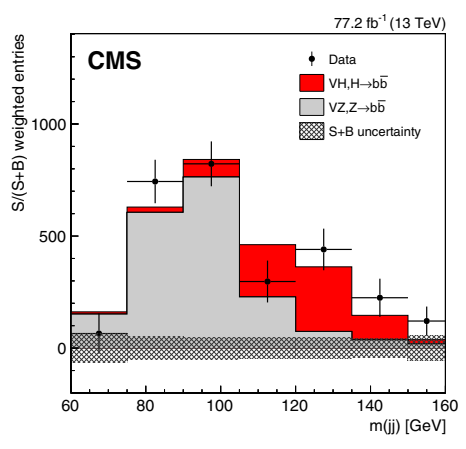

Figure 12. Dijet invariant mass distribution for events weighted by $\mathrm{S} /(\mathrm{S}+\mathrm{B})$ in all channels combined in the 2016 and 2017 datasets. The weights are derived from a fit to the dijet mass distribution [15].

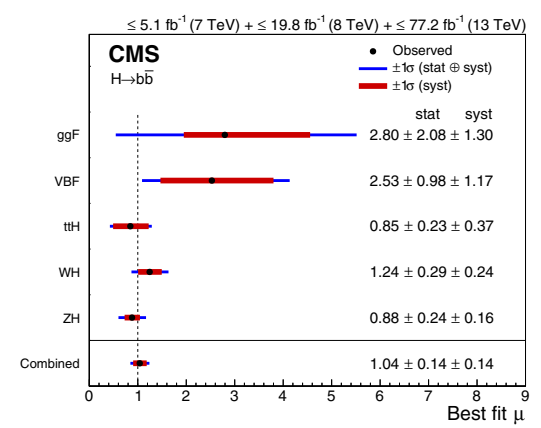

Figure 13. Best-fit value of the signal strength for Higgs boson decaying to pair of bottom quarks, for the five individual production modes considered, as well as the overall combined results [15].

\section{Higgs boson decay to tau lepton pair}

The Higgs boson decays to a tau lepton pair with the second largest branching fraction among the fermionic Higgs boson decays. In the analysis reported here [16] the cross section for a Higgs boson decaying to a tau lepton pair was measured by the CMS Collaboration. The STXS framework was used to split the cross section measurement by the production and decay modes. The neural network classification was used to categorize the events. Four different tau pair final states were considered: $e \mu, e \tau_{\text {had }}, \mu \tau_{\text {had }}$ and $\tau_{\text {had }} \tau_{\text {had }}$. A deep neural network method was used to identify the bottom quark jets, while the hadron-plus-strip algorithm was used for the reconstruction of tau leptons. The signal is present in the highest bins of the distribution in Fig. 14. The observed significance was measured to be 4.7 standard deviations, while the expected significance is estimated at 6.6 standard deviations. The inclusive 
cross section times the branching fraction of Higgs boson decay to tau pair is measured to be $\sigma_{\text {incl }} \times B(H \rightarrow \tau \tau)=2.56 \pm 0.46$ (stat.) \pm 0.34 (syst.) pb. When split into a production mode, the cross section corresponding to a gluon-gluon fusion merged with a $b \bar{b}$ plus Higgs boson production is measured to be $\sigma(g g \rightarrow H, b b H) \times B(H \rightarrow \tau \tau)=1.11 \pm 0.81$ (stat.) \pm 0.78 (syst.) $\mathrm{pb}$, while the VBF plus $\mathrm{V}(\mathrm{jj}) \mathrm{H}$ has a cross section of $\sigma(V B F+V(q q) H) \times B(H \rightarrow \tau \tau)=$ $0.34 \pm 0.08$ (stat.) \pm 0.09 (syst.) pb. The measured cross sections are in a good agreement with SM predictions, within the uncertainties.

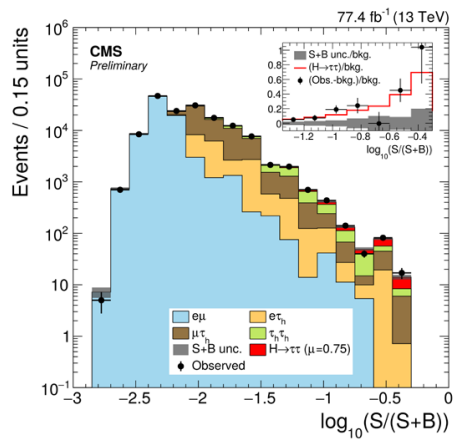

Figure 14. Distribution of the decimal logarithm of the signal purity after the fit to the data, evaluated in the bins of the signal categories used to extract the results. The signal and the background contributions from each decay channel are stacked on top of each other [16].

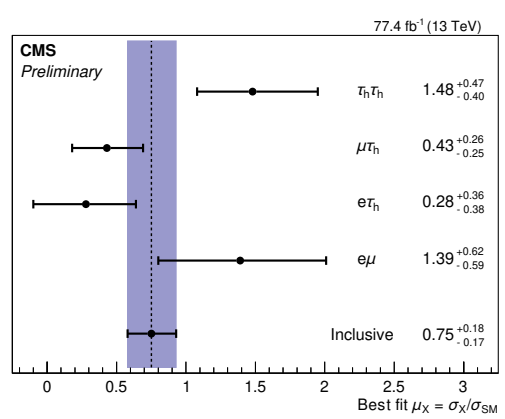

Figure 15. Results of the maximum likelihood fit to the data, for a model with one signal strength parameter, as discussed above, split by final state and inclusively, for all final states combined [16].

\section{Higgs boson decay to charm quarks}

The Higgs boson decaying to a pair of charmed quarks is searched for in this analysis [17] of the CMS Collaboration. This decay has around 20 times smaller branching fraction than the Higgs boson decay to a pair of bottom quarks and there is a larger contribution of the vector boson plus light flavour jets background. The search is performed considering associated production of a Higgs boson to a vector boson, being either a $\mathrm{W}$ or $\mathrm{Z}$ boson. The leading backgrounds are vector boson production associated with jets and also the $t \bar{t}$ process. Two distinctive topologies were considered in this analysis: the "resolved jet" topology in which the Higgs boson candidate is reconstructed from a two distinct charm quark jets and the "merged-jet" topology, in which the two charm quark jets are reconstructed as a single jet. The former focuses on the Higgs boson candidates with a low transverse momentum, while the latter performs better for the Higgs boson candidates with a high transverse momentum. The central feature of this search is the identification of a charm quark jets. Advanced machine learning techniques are used for the charm-jet identification. The merged-jet topology makes use of jet substructure information to further suppress the backgrounds. The performance of the charm-jet identification is presented in Fig. 16. A three different final states were considered, with either zero, one or two leptons. The signal was extracted using a binned likelihood fit in the two categories ("resolved" and "merged") and then combined. The observed (expected) $95 \%$ CL upper limit on the signal strength for VH production where the Higgs boson decays to a pair of charm quarks is presented in Fig. 17 and in the combination of the 
two analysis yields $\mu=70\left(37_{-11}^{+16}\right)$. This result is the most stringent direct limit on the $H \rightarrow c c$ cross section times branching fraction.

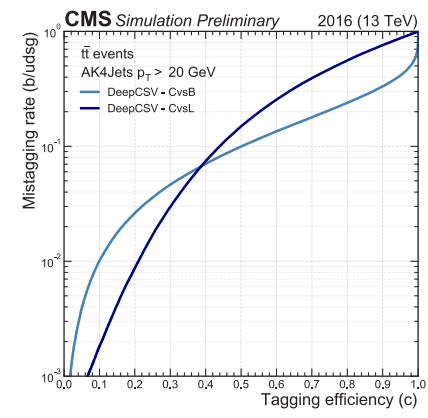

Figure 16. The two curves showing separately the tagging efficiency and the corresponding bottom jet and light jet mistag rate. The jets with $p_{T}>20 \mathrm{GeV}$ and clustered using the AK4 algorithm have been considered from a $t \bar{t}$ plus jets MC sample before the application of any data-to-simulation reshaping [17].

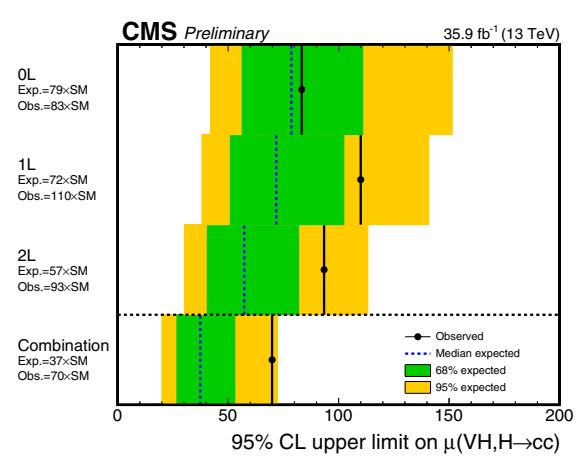

Figure 17. The 95\% confidence level upper limits on $\mu$ for the $\mathrm{VH}(H \rightarrow$ $c c$ ) process from the combination of the resolved-jet and merged-jet analyses in the different channels $(0 \mathrm{~L}, 1 \mathrm{~L}$ and $2 \mathrm{~L})$ and combined [17].

\section{VBF Higgs boson decay to invisible particles}

The analysis [18] of a Higgs boson produced via the VBF production mode and decaying to invisible particles is performed by the CMS Collaboration. In the SM, the Higgs boson decays invisibly only through the decay to four neutrinos, via a pair of $\mathrm{Z}$ bosons. The rate for the invisible decays of a Higgs boson may be enhanced in the context of several BSM scenarios, including those in which the Higgs boson acts as a portal to dark matter (DM) [19-21]. In the targeted VBF events, a Higgs boson is produced along with a two jets that exhibit a large separation in pseudorapidity $\left(\left|\Delta \eta_{j j}\right|\right)$ and a large dijet invariant mass $\left(m_{j j}\right)$. This analysis optimally exploits the distinctive kinematic features of the VBF topology by fitting the shape of the $m_{j j}$ distribution. The observed and the expected 95\% CL upper limits on $\left(\sigma / \sigma_{S M}\right) \mathrm{B}(\mathrm{H} \rightarrow \mathrm{inv})$ for a partial combination based either on the $7+8$ or $13 \mathrm{TeV}$ data as well as their combination is shown in Fig. 18. The results were interpreted also in the context of a Higgs-portal model of DM interaction, where the most stringent limits for $m_{\chi}$ smaller than 18(7) GeV, assuming a fermion (scalar) DM candidate, were provided, as shown in Fig. 19. These limits are computed at the $90 \%$ CL so that they could be compared with those from the direct detection experiments such as XENON1T [22], LUX [23], PandaX-II [24], CDMSlite [25], CRESST-II [26] and CDEX- 10 [27] which provide the strongest constraints in the $m_{\chi}$ range probed by this search.

\section{Higgs boson decay to muon pair}

The analysis [28] of a Higgs boson decaying to a pair of muons is performed by the CMS Collaboration. There is a small expected branching fraction for a Higgs boson decay to a pair of muons, having a value of $2.17 \times 10^{-4}$. The primary production mechanisms are the 


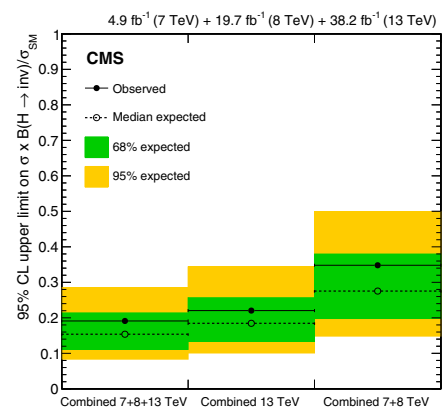

Figure 18. Observed and expected 95\% $\mathrm{CL}$ upper limits on $\left(\sigma / \sigma_{S M}\right) \mathrm{B}(\mathrm{H} \rightarrow$ inv $)$ for partial combinations based either on $7+8$ or $13 \mathrm{TeV}$ data and combination, assuming SM production cross sections for a Higgs boson mass of $125.09 \mathrm{GeV}$ [18].

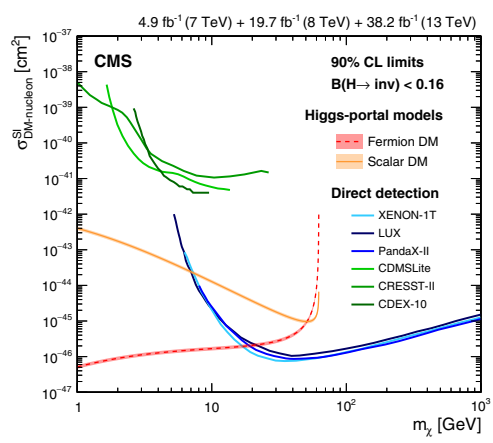

Figure 19. The 90\% CL upper limits on the spin-independent DM-nucleon scattering cross section in Higgs-portal models, assuming a scalar (solid orange) or fermion (dashed red) DM candidate [18].

VBF and ggH processes. To suppress the Drell-Yan, single top and $t \bar{t}$ backgrounds, boosted decision trees were used. An iterative procedure is applied in order to optimize the event categories, based on the sum of a signal events squared, over the number of background events. A total of 15 categories were utilized in this analysis. A maximum likelihood fit to the dimuon invariant mass was perfomed, as shown in Fig. 20. The analysis of the 2016 data yielded an observed (expected) upper limit on the Higgs boson to dimuon decay of 3.0(2.5) times the SM, while combined with the 7 and $8 \mathrm{TeV}$ data the observed(expected) upper limit is 2.9(2.2) times the SM expectation at 95\% confidence level, as shown in Fig. 21. Assuming the SM production cross sections for a Higgs boson, the observed upper limit on the Higgs boson branching fraction to two muons is $6.4 \times 10^{-4}$.

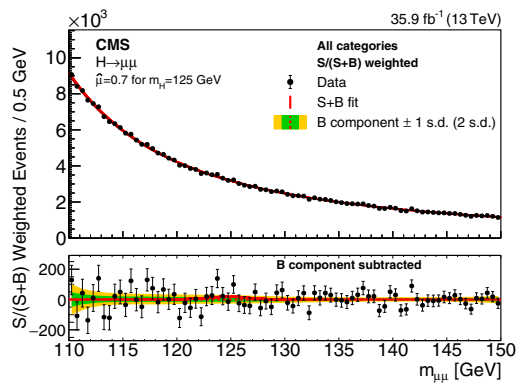

Figure 20. The data and weighted sum of signal-plus-background fits to each category. Events are weighted according to the expected ratio of signal to signal-plus-background in the category to which they belong [28].

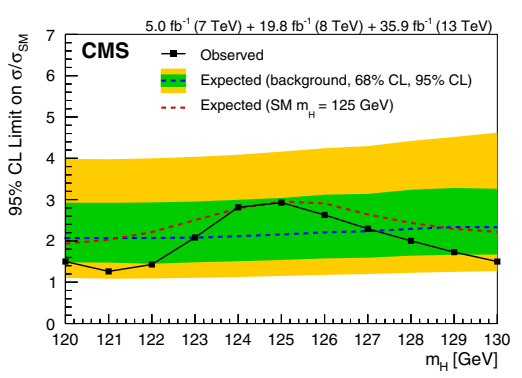

Figure 21. The 95\% CL upper limit on the signal strength modifier, $\mu$, in the region around the Higgs boson mass for the combination of the 7,8 , and $13 \mathrm{TeV}$ data together with the expected limit obtained in the background only and signal-plus-background hypothesis for the Higgs boson with $m_{H}=125 \mathrm{GeV}$ [28]. 


\section{Higgs boson pair production}

A search for Higgs boson pair production using the combined results from four final states: $b b \gamma \gamma, b b \tau \tau, b b b b$ and $b b V V$, where $\mathrm{V}$ represents a $\mathrm{W}$ or $\mathrm{Z}$ boson, is performed by the CMS Collaboration using the data collected in 2016 [29, 30]. The analysis of a Higgs boson self coupling represents an independent test of the SM and provides an access to the shape of the Higgs scalar field potential. It also allows searching for Higgs boson trilinear couplings by measuring the $\mathrm{HH}$ final state. There are heavy BSM resonances also predicted to decay to the $\mathrm{HH}$ final state. The non-resonant Higgs boson pair production is considered, using the Effective Field Theory (EFT) approach with five couplings: $y_{t}, \lambda_{H H H}, c_{2}, c_{2 g}$ and $c_{g}$, with $k_{\lambda}=\lambda_{H H H} / \lambda_{S M}$ and $k_{t}=y_{t} / y_{S M}$ defined. The resulting limits are reported in Fig. 22. The resonant signal that is searched for is represented by either a CP-even particle of spin- 0 (radion) or spin-2 (graviton) with a width that is much smaller than the detector resolution for the whole range under study. For the resonant production mechanism, the upper exclusion limits at $95 \% \mathrm{CL}$ are obtained for the production of a narrow resonance with masses ranging from 250 to $3000 \mathrm{GeV}$.

\section{Higgs boson couplings at HL-LHC}

The extrapolation studies for a Higgs boson coupling combination at $3000 \mathrm{fb}^{-1}$ have been performed by the ATLAS and CMS Collaborations [31]. The combined minimum negativelog-likelihoods are presented in Fig. 23. The 68\% confidence intervals for $k_{\lambda}$ are $0.52 \leq k_{\lambda} \leq$ 1.5 and $0.57 \leq k_{\lambda} \leq 1.5$ with and without systematic uncertainties, respectively. The second minimum of the likelihood is excluded at $99.4 \%$ CL. In addition to Higgs BSM searches, a substantial amount of parameter space (and masses) will be covered with the physics program at HL-LHC.

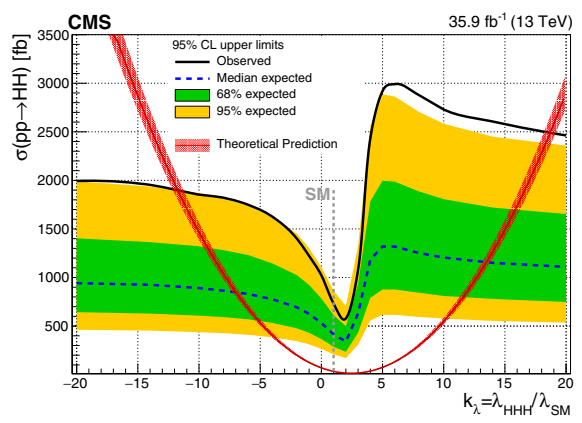

Figure 22. The expected and the observed $95 \%$ CL upper limits on the $\mathrm{HH}$ production cross section as a function of the $k_{\lambda}$ parameter [29].

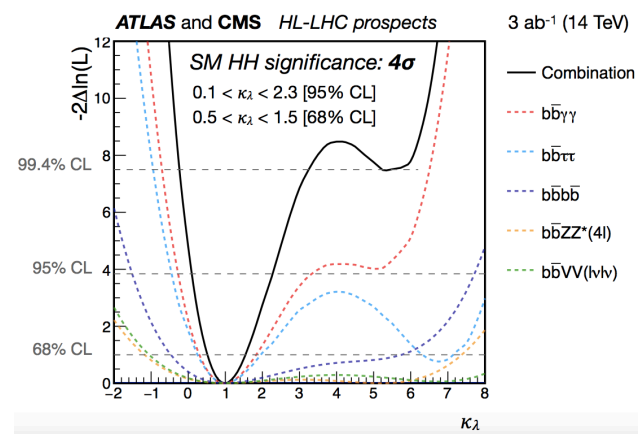

Figure 23. The minimum negative-loglikelihood as a function of $k_{\lambda}$, calculated by performing a conditional signal plus background fit to the background and SM signal [31].

\section{Conclusions}

The Higgs boson discovery in 2012 was a major event in high-energy physics. The properties of the Higgs boson are being characterized, in order to explore the electroweak symmetry 
breaking mechanism in the SM. The Higgs boson mass, spin-parity and width measurement were performed with unprecedented precision. The Higgs boson was observed separately in $\gamma \gamma, \mathrm{ZZ}, \mathrm{WW}, \mathrm{bb}, \tau \tau$ and $\mathrm{ttH}$ production modes. The inclusive and differential Higgs boson production cross section measurements were performed and new approaches have been adopted, like the STXS framework. Rare Higgs boson decay channels have been studied, like the Higgs boson decay to a muon pair, to a pair of charm quarks and the Higgs boson self-coupling. No deviations from the SM prediction have been observed. At the HL-LHC a significant improvement of the measurement precision of the Higgs boson couplings is expected.

\section{References}

[1] The ATLAS Collaboration, Phys. Lett. B 716, 1 (2012).

[2] The CMS Collaboration, Phys. Lett. B 716, 30 (2012).

[3] The CMS Collaboration, JHEP 06 (2013) 081.

[4] The CMS Collaboration, JINST 3 S08004 (2008).

[5] The CMS Collaboration, CMS-PAS-HIG-18-029 (2019), https://cds.cern.ch/record/ 2667225.

[6] LHC Higgs Cross Section Working Group Collaboration, "Handbook of LHC Higgs Cross Sections: 4. Deciphering the Nature of the Higgs Sector", doi:10.23731/CYRM2017-002, arXiv:1610.07922.

[7] The CMS Collaboration, JHEP 11 (2017) 047.

[8] The CMS Collaboration, PRD 99 (2019) 112003.

[9] The CMS Collaboration, Eur. Phys. J. C 75 (2015) 212.

[10] The ATLAS Collaboration, Phys. Rev. D 90 (2014) 052004.

[11] The CMS Collaboration, CMS-PAS-HIG-19-001 (2019), https://cds.cern.ch/record/ 2668684.

[12] The CMS Collaboration, PRL 120 (2018) 231801.

[13] The CMS Collaboration, CMS-PAS-HIG-18-019 (2019), http://cds.cern.ch/record/ 2649199.

[14] The CMS Collaboration, CMS-PAS-HIG-18-030 (2019), https://cds.cern.ch/record/ 2675023.

[15] The CMS Collaboration, PRL 121 (2018) 121801.

[16] The CMS Collaboration, CMS-PAS-HIG-18-032 (2019), https://cds.cern.ch/record/ 2668685.

[17] The CMS Collaboration, CMS-PAS-HIG-18-031 (2019), https://cds.cern.ch/record/ 2682638.

[18] The CMS Collaboration, Phys. Lett. B 793 (2019) 520.

[19] A. Djouadi, O. Lebedev, Y. Mambrini, and J. Quevillon, Implications of LHC searches for Higgs portal dark matter, Phys. Lett. B 709 (2012) 65.

[20] S. Baek, P. Ko, W.I. Park, and E. Senaha, Higgs portal vector dark matter: revisited, JHEP 05 (2013) 036.

[21] A. Djouadi, A. Falkowski, Y. Mambrini, and J. Quevillon, Direct detection of Higgs portal dark matter at the LHC, Eur. Phys. J. C 73 (2013) 2455.

[22] XENON Collaboration, Dark matter search results from a one ton-year exposure of XENON1T, Phys. Rev. Lett. 121 (2018) 111302.

[23] LUX Collaboration, Results from a search for dark matter in the complete LUX exposure, Phys. Rev. Lett. 118 (2017) 021303. 
[24] PandaX-II Collaboration, Dark matter results from first 98.7 days of data from the PandaX-II experiment, Phys. Rev. Lett. 117 (2016) 121303.

[25] SuperCDMS Collaboration, New Results from the Search for Low-Mass Weakly Interacting Massive Particles with the CDMS Low Ionization Threshold Experiment, Phys. Rev. Lett. 116 (2016) 071301.

[26] CRESST Collaboration, Results on light dark matter particles with a low-threshold CRESST-II detector, Eur. Phys. J. C 76 (2016) 25.

[27] CDEX Collaboration, Limits on light weakly interacting massive particles from the first $102.8 \mathrm{~kg}$ x day data of the CDEX-10 experiment, Phys. Rev. Lett. 120 (2018) 241301.

[28] The CMS Collaboration, PRL 122 (2019) 021801.

[29] The CMS Collaboration, PRL 122 (2019) 121803.

[30] The CMS Collaboration, Eur. Phys. J. C 79 (2019) 421.

[31] HL/HE WG2 group (Cepeda, M. et al.), Higgs Physics at the HL-LHC and HE-LHC, arXiv:1902.00134 [hep-ph] CERN-LPCC-2018-04. 